\title{
LEONID STORM FLUX ANALYSIS FROM ONE LEONID MAC VIDEO AL5OR
}

\author{
PETER S. GURAL \\ Science Applications International Corporation. 4001 N. Fairfax Drive, Suite 500, \\ Arlington, Virginia 22203, USA \\ E-mail: peter.s.gural@saic.com \\ and \\ PETER JENNISKENS \\ The SETI Institute, NASA Ames Research Center, Mail Stop 239-4, Moffett Field, \\ California 94035, USA \\ E-mail: pjenniskens@mail.arc.nasa.gov
}

(Received 31 May 2000; Accepted 16 August 2000)

\begin{abstract}
A detailed meteor flux analysis is presented of a seventeen-minute portion of one videotape, collected on November 18, 1999, during the Leonid Multi-instrument Aircraft Campaign. The data was recorded around the peak of the Leonid meteor storm using an intensified CCD camera pointed towards the low southern horizon. Positions of meteors on the sky were measured. These measured meteor distributions were compared to a Monte Carlo simulation, which is a new approach to parameter estimation for mass ratio and flux. Comparison of simulated flux versus observed flux levels, seen between 1:50:00 and 2:06:41 UT, indicate a magnitude population index of $r=1.8 \pm 0.1$ and mass ratio of $s=1.64 \pm 0.06$. The average spatial density of the material contributing to the Leonid storm peak is measured at $0.82 \pm 0.19$ particles per square kilometer per hour for particles of at least absolute visual magnitude +6.5 . Clustering analysis of the arrival times of Leonids impacting the earth's atmosphere over the total observing interval shows no enhancement or clumping down to time scales of the video frame rate. This indicates a uniformly random temporal distribution of particles in the stream encountered during the 1999 epoch. Based on the observed distribution of meteors on the sky and the model distribution, recommendations are made for the optimal pointing directions for video camera meteor counts during future ground and airborne missions.
\end{abstract}

Key Words: Clustering, flux, fragmentation, Leonids 1999, meteor, meteoroid, optimal video pointing, satellite impact hazard, simulation 


\section{Introduction}

The spatial number density of particles in the Leonid meteor stream is of paramount concern to the U.S. Air Force and NASA, as well as other satellite operators. The 1999 Leonid shower was anticipated to cause several orders of magnitude increase in the population of fast meteoroids for satellites exposed to the shower (Jenniskens et al., 1998). Near realtime reporting systems were set up globally to provide immediate access to the severity of the meteor storm during the period of the shower (Brown et al., 2000). The NASA and USAF sponsored Leonid Multi-InstrumentAircraft Campaign participated in this effort by providing near real-time meteor counts to satellite operators worldwide (Jenniskens et al., 2000a).

An accurate calibration of fluxes and the measurement of the meteoroid size distributions, however, demands an after-the-fact analysis, involving the data processing of video tapes collected from eight cameras taken on three consecutive nights of data gathering (Jenniskens et al., 2000b). Such work is also needed to reveal size-dependent dispersions in the shower and possible non-statistical fluctuations that can be traced back to particles breaking up in space (Jenniskens and Butow, 1999).

This paper presents an analysis of a small time interval from just one of the tapes recorded. This analysis includes a comparison to a meteor simulation model in order to derive key meteor shower parameters such as mass index and spatial number density. In comparison, such parameters have in the past been determined through measured counts and magnitude binning with correction factors applied to account for various geometric effects (Koschack, 1990a; 1990b). Using a simulation to attempt to match observed flux levels for the derivation of shower characteristics, is an alternative approach and one that is new for the meteor community. Monte Carlo techniques have been used in the past to study the observational influences on both the magnitude distribution index and zenith hourly rate corrections (e.g. Van der Veen, 1986; Arlt, 1998). Our work significantly expands on the technique by directly estimating meteor shower parameters via simulation and measurement comparisons and by including higher fidelity modeling. The advantages are that in the process of improving the fidelity of the simulation to match observations, magnitude loss models and instrument characteristics are refined that help to characterize the visibility and flux of the shower under different viewing conditions. Based on these models, recommendations for best viewing directions can be made for future data collection missions using similar techniques. The principal goal of this work, however, is to estimate the actual spatial number density of meteoroids during the peak flux of the 1999 Leonid storm. 


\section{Measured Data Set}

The Leonid MAC mission involved two aircraft, ARIA and FISTA, flown in tandem with multiple instruments onboard for collecting data on meteor tracks and trains (Jenniskens et al., 2000). The video record studied here was taken onboard the Advanced Ranging and Instrumentation Aircraft (ARIA), looking due south at low elevation. It was designated AL50R (ARIA Low elevation 50mm Right). The flight path for the night of maximum, November 18,1999, took the aircraft on a east-northeasterly path from Israel to the Azores over a period of eight hours. The second tape from the night of November 18 was studied in detail because it contained the imagery during the time of peak storm flux around 2:00 UT. This part was also documented by visual inspection of the video record, which provided a baseline for subsequent analysis.

The imaging camera consisted of a low f-number objective of $50 \mathrm{~mm}$ focal length $\mathrm{f} / 1.4$ coupled to an AEG multi-channel plate secondgeneration image intensifier from standard military use "night-goggles". The output of the intensifier was recorded with a Sony CCD-TRV65 Handycam Hi-8 camcorder by macro focusing on the back phosphor plate of the intensifier. The camera was pointed through one of the ARIA's BK7 optical quality glass windows whose transmission characteristics did not affect the sensitivity of the camera in the spectral region of interest. The camera performed similarly to the systems described in Jenniskens (1999a), with slightly worse coupling of the higher resolution intensified star images with the relatively low camcorder image resolution and a slightly different distribution of noise levels across the intensifier.

\section{Data Analysis Procedure}

The data were recorded on $\mathrm{Hi}-8 \mathrm{~mm}$ videotape and required digitization prior to performing the various analyses necessary for flux estimation. A Scion Corporation LG-3 video frame grabber installed in a Macintosh $\mathrm{G} 3 / 400 \mathrm{MHz}$ computer formed the basis of the imagery analysis hardware. This system was capable of capturing every frame in the video record at full resolution in real time.

To obtain an accurate estimation of measured flux above the imaging system's limiting magnitude it was decided to avoid using the automated meteor detection features of the software "MeteorScan" written by one of the authors (Gural, 1999a). Although this software typically provides detection efficiencies approaching $80 \%$ of the meteors on a single pass of videotape, the software can suffer from selection effects based on short path lengths of meteors and low detection probability near the noise limit of the imagery. For the imager chosen, many of the lowest elevation meteors have very short streaks which are difficult to identify as meteors for the line detection and moving target detection algorithms in the 
software. Thus an interactive human detector was employed which has been found to be very efficient at motion detection of very faint objects in video.

The "MeteorScan" software was modified to first digitize several minutes of imagery and then play it back in one second movie loops for visual analysis. This required some special purpose software just to digitize the data since writing video streams to disk was found to be slower than real time. Thus four separate passes of the tape were required with $25 \%$ time overlap of one-second sequences. These were later synched up, via a spatial image correlation, on the changes in the seconds character of the superimposed time stamp.

Each second of video was replayed continuously so the user had many looks at the imagery to determine if a meteor existed. In addition to the raw imagery display, the user could also examine the difference frames, where a moving track stands out more clearly above the noise. Once a track was identified, the user would position a cursor over the beginning and ending points, mouse clicking to define the meteor start and stop positions. The track would be marked on the screen so that during the next one second of time overlapped video, the user would not repeat the count of the same meteor. After sequencing through roughly fifty seconds of video or one CD's worth, the selected tracks would be reprocessed in higher fidelity to refine estimations of begin and end points, apparent angular speed, integrated intensity, and frame of peak brightness. The refinement was done by applying a matched filter along the meteor's path and varying four track parameters in a downhill simplex search for the maximum likelihood ratio. This provided the best fit of a propagating line to the video sequence of images.

Only those meteors that had an end point within the camera's field of view were counted thus defining the effective field of view (Jenniskens, 1999a). It is necessary to calibrate the field of view to convert pixel coordinates for the end points to azimuth and elevation in an earth inertial coordinate system centered on the imager. This was done at the beginning of each period of fifty seconds using several known stars in the field and applying the astrometry algorithms of Steyaert (1990). Inputs to this process were the ARIA's position in latitude, longitude, and height obtained off recorded logs from the Global Positioning System. For the AL50R, the field of view was found to be stable to within plus or minus one degree for the time interval studied. The azimuth and elevation of the image center (Figure 1 and 2) wavered little except for a aircraft turn of three degrees at 1:54 UT. Thus the elevation was deemed stable enough to be able to bin meteor counts in elevation without having to calibrate each individual image frame. 


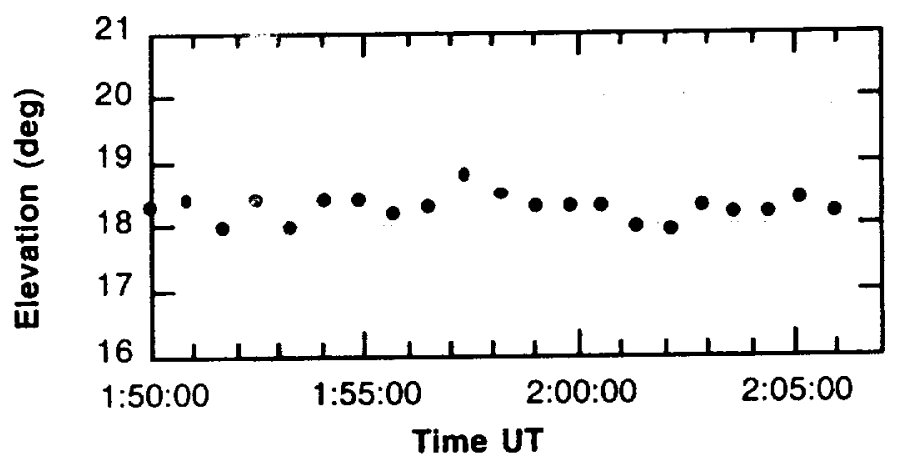

Figure 1. Image center elevation as a function of time. Note the relatively stable imager pointing during the data analysis interval.

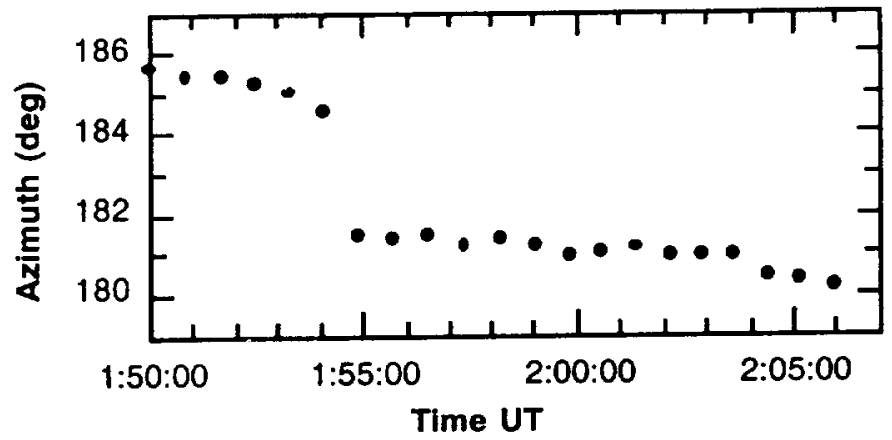

Figure 2. Image center azimuth as a function of time. The ARIA aircraft executed an azimuth adjustment of three degrees at 1:54 UT.

\section{Results}

\subsection{SPATIAL DISTRIBUTION}

In total there were 1512 meteors identified on the AL50R videotape from 1:50:00-2:06:41 UT of which 99.2\% were Leonids. A plot of the end point positions in azimuth and elevation (Figure 3 ) after the aircraft turn 
shows the elevated counts near the horizon as one looks through a larger atmospheric volume. This high count of meteors at low elevation is a key advantage to flying at high altitude. This was first recognized and came as a surprise during the 1998 Leonid MAC mission (Jenniskens, 1999b).

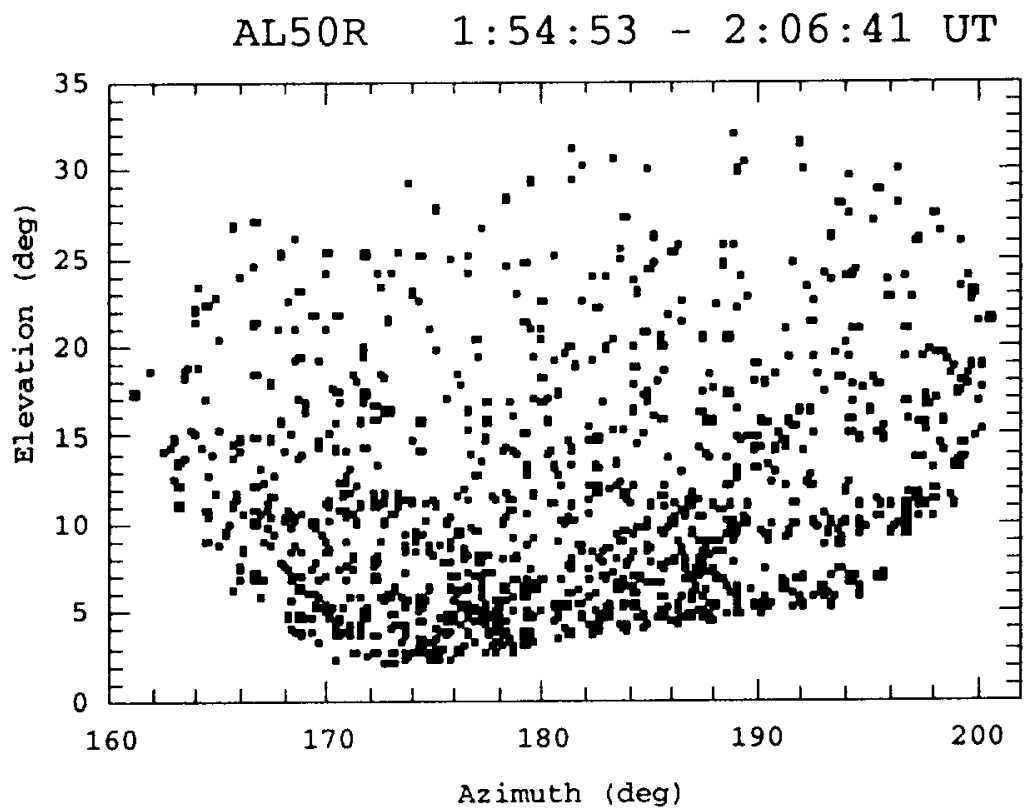

Figure 3. End point positions of AL50R meteors recorded between 1:54:53 UT and 2:06:4I UT.

The response of the imager is fairly uniform across in azimuth over almost the entire field of view. This bodes well for not having to correct for imager sensitivity or lens vignetting in the later analysis. A lack of meteors seen in the lower right corner can be accounted for by the interference from the superimposed time stamp that blocked the visibility of meteors from the user in that portion of the field of view. The sloped edge of the field of view at the lowest elevation angles is a result of the camera mounting alignment and pitch up of the aircraft relative to the local horizon.

\subsection{TEMPORAL DISTRIBUTION}

Examination of the raw video counts versus time during the time of the storm's peak, shows a fairly flat flux profile (Figure 4) with activity in the AL50R field of view averaging 80 meteors per minute with several bursts 
of activity as high as 110 meteors per minute. At the ten second resolution scale (Figure 5), it is apparent that a relatively constant background level seems to be punctuated with flux rates that can nearly double over very short observation times. These possible waves of meteors deserve attention in future analysis of the remaining video records from the other cameras. Please note that the flux estimation made in the latter part of this paper will represent an average level over the period of time analyzed and should include the caveat that waves of meteoroids can easily raise the spatial number density on a short time basis.

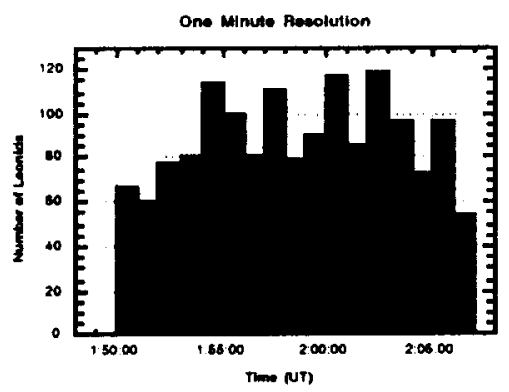

Figure 4. Raw Leonid video counts versus time at a one minute resolution.

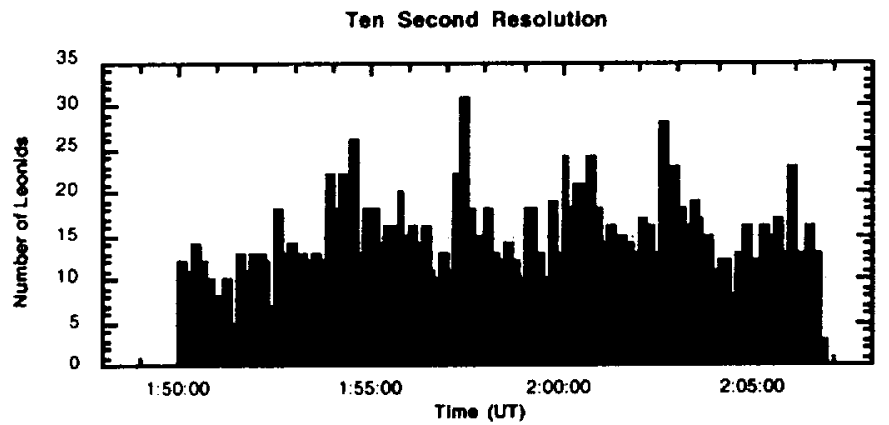

Figure 5. Raw Leonid video counts versus time at a ten second resolution.

\subsection{METEOR TRAIL PROPERTIES}

It was first thought that the larger distances to those Leonids near the horizon would result in the detection of a smaller part of the meteor trajectory. The issue is that the meteor's light curve has a brightening and fading temporal response and the dimmer begin and end points would be invisible due to extinction and distance losses. Thus we would expect the 
trail lengths to be more foreshortened than theoretically predicted. We examined the observed trail lengths and compared them to theoretical values based on typical Leonid beginning and end heights. The heights chosen were based on extrapolation of photographic beginning heights suggesting a beginning height of only $108 \mathrm{~km}$. whereas the typical end point of Leonids have been found to have a height of $95 \mathrm{~km}$ (Jenniskens $e t$ al., 1998; Brown et al., 2000). Of course, beginning and end heights are derived from meteors observed at short range (high elevation angle) so relating the low elevation observations to the shorter-range measurements of the past can be problematic.

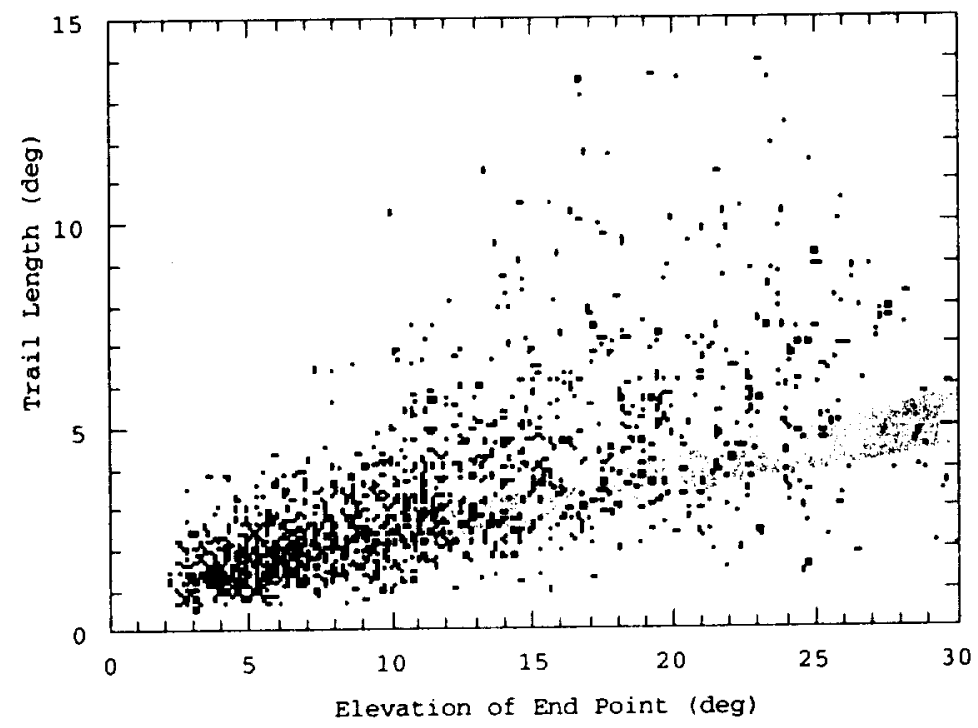

Figure 6. Visible meteor trail lengths versus elevation compared to typical Leonids with beginning heights of $108 \mathrm{~km}$. and ending heights of $95 \mathrm{~km}$. for the AL50R at the time of peak flux. The dots are measured trail lengths from the video and the gray area represents expected values given average Leonid parameters over the entire field of view.

From the measured positions of the meteor begin and end points in image coordinates and the calibration of the field of view in stellar coordinates, one can determine radiant distance and the measured visible trail length presented to the low elevation imager (Figure 6). A predicted set of trail 
lengths can be defined by using an average beginning height of $108 \mathrm{~km}$, an end height of $95 \mathrm{~km}$, and solving for the geometric lengths presented to all positions in the AL50R field of view for the appropriate Leonid radiant position. This was done for the ARIA's position at 2:00 UT on November 18,1999 . It can be seen that the measured meteor trail lengths (dots) fall in and around the average predicted Leonid values (gray area). The spread in measured values is caused by both a distribution of begin heights and missing components of the fainter tracks due to distance.

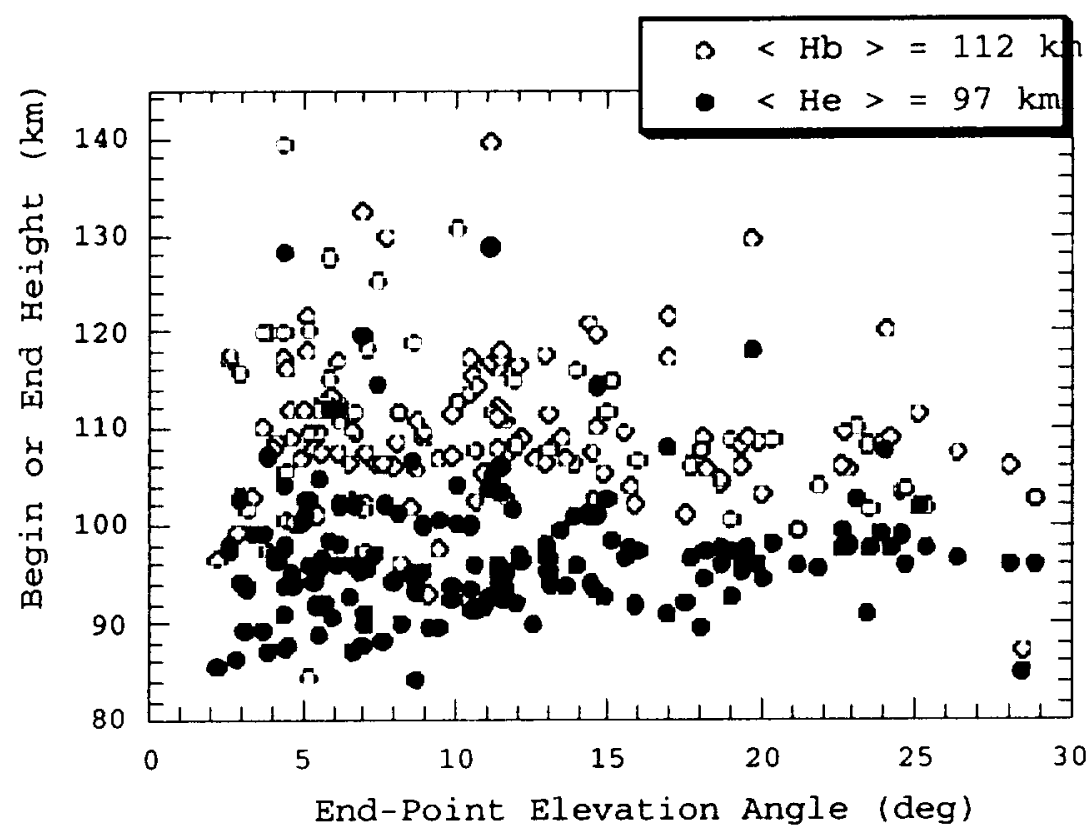

Figure 7. Leonid beginning and ending heights based on single station estimates showing similarity to published values and no apparent trend with elevation angle. Shown is a set of 172 meteors whose angular velocities were measured more precisely representing all meteors observed between 1:59:48 and 2:01:19 UT.

To double check the beginning and end heights chosen, one can use a single station formulation for $\mathrm{H}_{c}$ and $\mathrm{H}_{b}$, since we know the entry velocity for Leonids $(71 \mathrm{~km} / \mathrm{sec})$, the sensor altitude $(11.0 \mathrm{~km})$, the distance of the track from the radiant, and the apparent angular velocity $\omega$. We first reevaluated the angular velocity more accurately for all meteors over a period 
of one and a half minutes centered near 2:00 UT, and computed the heights using a ground based formula (Gural, 1999b) corrected for high altitudes. $R$ is the earth's radius and $H_{\text {sensor }}$ the sensor altitude.

$$
H=\left[\left(R+H_{\text {sensor }}\right)^{2}+2\left(R+H_{\text {sensor }}\right)|q| \sin (\text { Elevation })+|q|^{2}\right]^{12}-R
$$

where:

$$
|\mathrm{q}|=\mathrm{V}_{\infty} \sin (\text { Radiant Distance ) / } \omega(\mathrm{rad} / \mathrm{sec})
$$

The formula is valid for any point along the meteor's track but suffers from larger errors as the elevation decreases $( \pm 2 \mathrm{~km}$ at 25 degrees versus $\pm 5 \mathrm{~km}$ at 5 degrees) for the given angular velocity accuracy $( \pm 0.0065$ $\mathrm{rad} / \mathrm{sec}$ limited by the video resolution). Nevertheless, the mean begin and end heights match very closely to the values suggested from other published results (Figure 7). There is also no obvious trend in the data as a function of elevation angle, although the errors associated the low elevation single station measurements make this somewhat inconclusive.

\section{Flux Modeling and Simulation}

A Monte Carlo meteor influx simulation was developed for comparison with the observations. The objective was to estimate the spatial density of meteors from the 1999 Leonid storm and to determine the optimal camera pointing directions for future data collection missions.

The technique relates the raw observed meteor counts to a statistically derived sample of counts based on a few parameterized shower variables. The statistical flux levels are obtained by ray tracing simulated meteor paths through realizable geometry and magnitude losses, and counting them over a restricted field of view. This method avoids some of the pitfalls inherent in adjusting measured meteor counts for perception and low elevation observations that have been used until now. However, the method requires reasonably good models for magnitude losses from atmospheric extinction, distance fading, and apparent angular speed. Although the ARIA and FISTA low elevation video data were collected at an altitude with little to no extinction loss, the simulation includes such losses to better model observations that could be potentially collected at any altitude.

The simulation is initialized with a uniformly distributed set of randomly positioned meteoroids in a three dimensional cylinder (Figure 8 ). The region in space containing the meteoroids is aligned with its long axis parallel to and centered on the observer's radiant vector $\underline{\mathbf{r}}$ with the orthogonal dimensions representing the cross-track orientation. All the particles are assumed to move in parallel along the direction $\underline{\mathbf{r}}$ with entry 
velocity $V_{\infty}$. The cross-section of the particle swarm is taken large enough to encompass every look direction of an all-sky sensor that intersects a sphere of radius beginning height plus Earth's radius. This defines a meniscus that extends to the observer's zero elevation horizon in all azimuth directions, and is assured to be intersected by meteoroids in all possible places independent of radiant elevation. The meteoroids are assumed to travel in straight lines without deceleration along the atmospheric path. Zenith attraction is not accounted for but can be neglected in the case of the Leonids with high entry velocities at medium radiant elevations. Another factor ignored for this simulation was that the magnitude is dependent on the entry angle of the meteor. However, for radiant elevations above ten degrees the curvature of the atmospheric cap is so slight as to cause little variation in entry angle across the sky at a given point in time. Since the period of analysis covered only a degree change in radiant elevation angle it was deemed unnecessary to model the entry angle effect at this point.

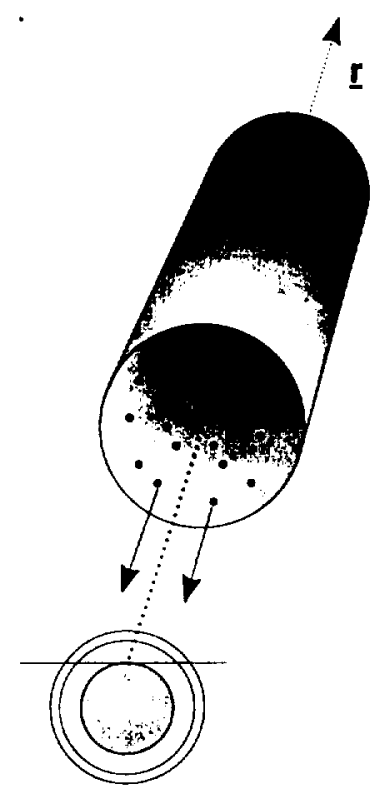

Figure 8. Geometric model of the meteor simulation developed for the analysis of observed flux and prediction of best pointing of image intensified video cameras. In the lower portion of the figure, the inner circle represents the earth's surface surrounded by two concentric circles at the meteor begin and end heights. The cylinder is a three-dimensional volume in space containing randomly distributed particles whose central axis is aligned with the observer's radiant vector I (dotted line). Each particle moves with the same velocity in parallel paths indicated by the arrows pointing towards the Earth. 
The meteoroids are randomly assigned : magnitude referenced to a particle ablating at the zenith 100 kilometers : ve the earth's surface. The magnitude distribution follows from : user-specified magnitude distribution index $(r)$ and the classic number

$$
\mathrm{n} \text { (magnitude) }=\mathrm{N} \mathrm{r}^{\text {(magni: }}
$$

such that $l m$ is defined as the limiting magnit: $:$ and $N$ as the total number of meteoroids of magnitude $\mathrm{lm}$. Each $\mathrm{m}$ oroid is ray traced to the intersection with the atmosphere and assign a random magnitude from the distribution before any losses are accou $d$ for. The value of limiting magnitude used in the simulation was based : the sensor's sensitivity and the $\mathrm{r}$-factor was allowed to vary to find the $\mathrm{bc}$ : it to the observations.

The meteoroid must pass two visibility cri! ia before being counted as "seen" by the simulated imaging system. "he first criteria, is that the meteor must be above the observer's hori. at some point along the visible portion of the meteor's path. For elevation count analysis defined in the previous section, only those $r$ zors were counted that had their endpoints visible in the camera's field a view. Thus for the purpose of the simulation, the end height was used as ine defining position for the first visibility test. As there can be two solu ins for that point in space, entry and exit from the atmospheric cap, the sition of first entry into the atmosphere is used in the test.

The second criteria required the meteor magnitude to exceed the limiting magnitude, after taking into account "ses for extinction, distance, and the meteor's apparent angular velocity The extinction loss, as a function of air mass " $X$ ", was based on a qu iratic fit to the tables found in Roth (1994, Appendix B). For a ground t: ad observer the expression used for visual magnitudes was:

$$
\Delta \mathrm{m}_{\mathrm{v}}=-0.003 \mathrm{X}^{2}+0.228 \mathrm{X}-0.225 \quad \mathrm{X}<35 \text { air masses }
$$

Alternative formulations for extinction have published, such as the model developed by Koschny and Zender :2000), and could easily be incorporated into future simulations

To obtain the extinction at higher observer iltitudes, it was decided to calculate the air mass at the new observer's height for each elevation angle and apply the ground-based extinction for the air mass. The air mass is given by integrating the density of the 1962 13.S. Standard Atmosphere along an elevation line from the observer posision to a point 50 kilometers in altitude. This is normalized by the intes al of air density from the ground to the zenith. Thus given the elevatio: and sensor height, the air mass $X$ was computed and the magnitude inss due to extinction $\Delta m_{v}$ 
determined. Extinction losses are normalized to airmass $=1.0$ (zenith). The airmass is in fact less than one for zenith observations at altitude. The loss as a function of elevation for ground based, mountaintop, and airborne altitudes shows the advantage of an airborne sensor (Figure 9). Clearly the extinction losses are far lower when imaging at the high altitude of an airborne mission and is a major contributor to the high meteor counts seen by the aircraft's low elevation cameras during the storm.

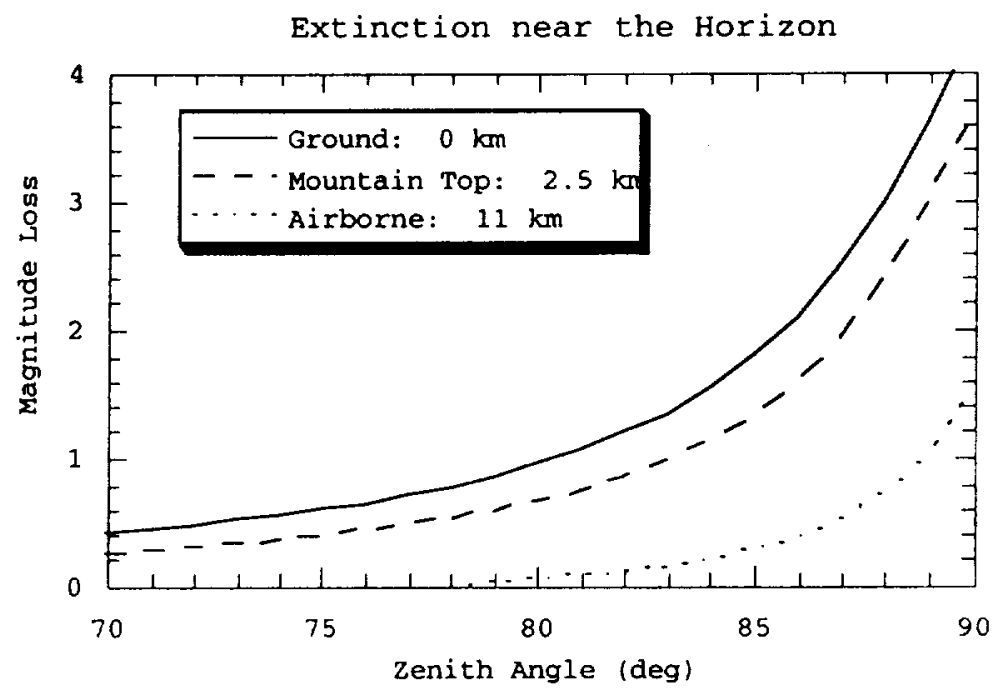

Figure 9. Extinction as a function of zenith angle for various sensor altitudes.

Magnitude losses for the meteor's distance from the observer beyond 100 kilometers is accounted for with the expression:

$$
\Delta \mathrm{m}=5.0 \log _{10}(\text { Distance in } \mathrm{km} . / 100)
$$

The distance loss is normalized for a distance of $100 \mathrm{~km}$, hence there is again a small magnitude gain near the zenith where the aircraft is less than $100 \mathrm{~km}$ from a meteor positioned directly overhead.

The final magnitude loss expression includes the effect of the apparent angular velocity " $\omega$ " of the meteor and the short time span it spends on a given pixel in the CCD camera. The loss is taken as (Jenniskens et al., 1998):

$$
\begin{array}{ll}
\Delta \mathrm{m}=2.5 \log _{10}\left(\omega_{\text {meteor }} / \omega_{0}\right) & \left(\omega_{\text {meteor }}>\omega_{0}\right) \\
\Delta \mathrm{m}=0 & \left(\omega_{\text {meteor }}<\omega_{\mathrm{o}}\right)
\end{array}
$$


where $\omega_{0}$ is the subtended angular size of the sky imaged by the pixel divided by the integration time of the pixel. The losses are taken as zero when the meteor stays within a single pixel during the entire integration period of a single video frame (1/60 $0^{\text {th }}$ of a second for interlaced video), otherwise there would be a net magnitude gain from blind application of the formula. The effect of angular velocity loss is to lower the counts of higher elevation meteors since they have a faster apparent angular velocity than those near the horizon.

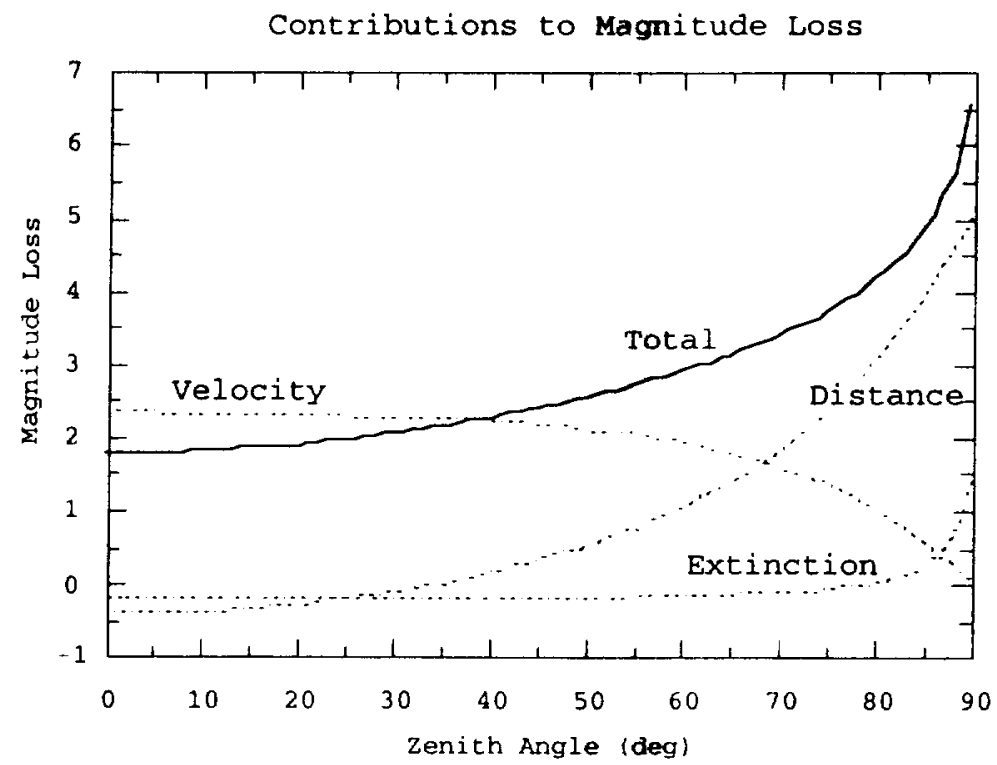

Figure 10. Total magnitude loss versus zenith angle for azimuth $180^{\circ}$ and Leonid radiant position as seen from ARIA at 2:00 UT November 18, 1999. The losses include the effects of extinction, distance, and meteor angular velocity.

The combination of these three loss terms (Figure 10) is added to the simulated meteor's initially assigned magnitude with the result compared to the limiting magnitude. If a simulated meteor is brighter than the limiting magnitude and appears above the observer's horizon it is declared as having been detected. Here, it is assumed the sensing system has unity response across the field of view. For a human observer it would be necessary to add a detection efficiency factor as a function of the off axis angle from the line-of-sight direction, and to consider detection along the entire luminous track of the meteor rather than just at the end point. For the AL50R imager, the response across the field of view will be considered flat 
but adjustments will be made to the sensor area due to clipping of the image in the comers and tilt of the imager with the horizon.

When all visibility criteria are passed, the meteor is counted and binned into an azimuth / elevation table with one degree resolution. One billion simulated meteors are examined producing an all-sky map of observable flux for a given set of r-factor, radiant coordinates, entry velocity, end height, limiting magnitude, $\omega_{o}$, and observer altitude. A final correction to normalize every bin to "meteors per square degree" is made by dividing each counting bin by the cosine of the elevation angle.

\section{Comparison of the Simulation with Observations}

\subsection{ESTIMATING THE SHOWER PARAMETERS}

To make an estimate of the spatial number density of meteoroids during the Leonid storm, it is necessary to determine an $r$-factor, the magnitude distribution index, which best matches the observed measurements. For the 1999 Leonids, results thus far for published visual data (Arlt et al., 1999), and a preliminary estimate for radar and video data (Brown et al., 2000), indicate an r-factor of 2.3 on the night of November 18, 1999. These all have been based on binning magnitude counts and either computing the mass ratio or magnitude distribution index.

The attempt in this paper is to determine the r-factor independently of magnitude estimates and rely on a simulation tool to match the observations. If the camera can be assumed to respond equally well over the entire field of view, then the variation in observed elevation counts can be compared to simulated levels by varying the r-factor until a good match is obtained. It was verified that the limiting magnitude did not vary below 20 degrees elevation by examining the sky background in the lower half of the image. The upper half did show a darker sky background, but the critical area for the fits to slope in the elevation counts is below 20 degrees so the uniform response assumption is valid in that region.

A plot of meteor counts (Figure 11) at each elevation for the AL50R observations was determined by averaging the counts across in azimuth as both the measurements and simulation show a weak flux dependence on azimuth for the southerly look direction. Aircraft pitch and roll were stable enough that angle-pointing variations of only plus or minus one degree occurred over the time period of interest. The averages were taken over three degree elevation swaths to smooth out the effects of sample size and platform pointing instability. The comparison of the measured points (dots) to the simulated curves (solid and dashed) for various $\mathrm{r}$-factors are shown. Based on the simulated results, the best $r$-factor found was $r=1.8$ \pm 0.1 . 


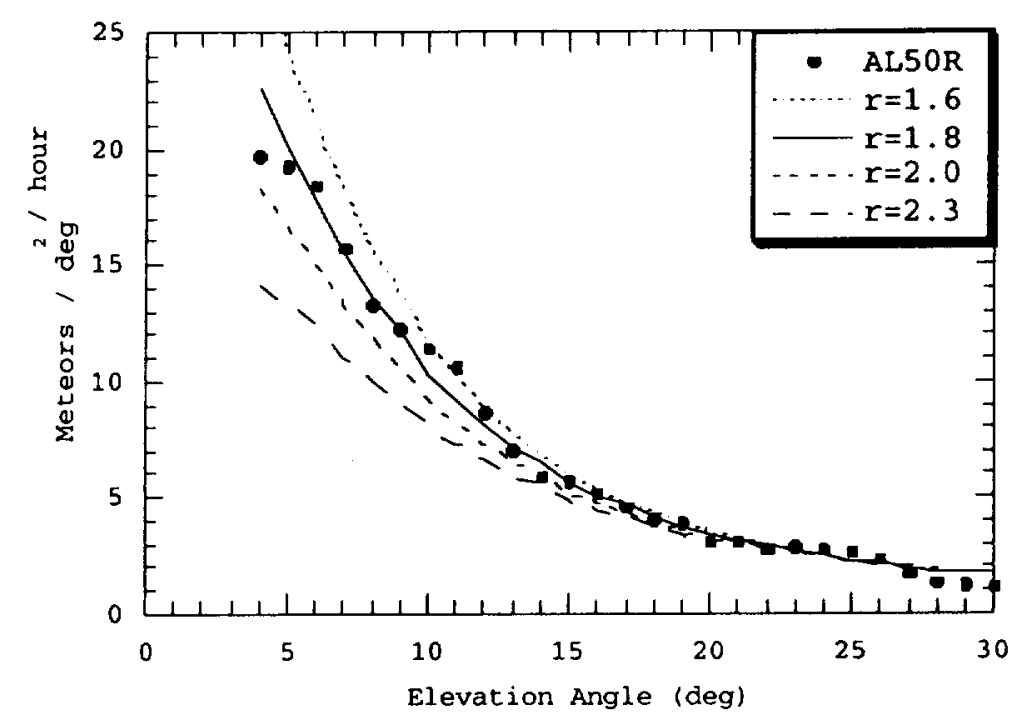

Figure 11. Measured meteor counts per square degree per hour compared to simulated counts for various r-factors. All curves have been normalized to equivalent flux at thirty degrees elevation.

The discrepancy in the $r$-factor with other published results is puzzling. We checked if the measurements on the ground may be affected by observing geometry and conditions. Using $r=1.8$ and determining the actual magnitude ratios that would have been observed by a video camera on the ground using the simulation tool, yielded an r-factor of 1.8 to 1.9 , depending on pointing. Thus the model shows that there is no distortion of the r-factor observed on the ground relative to the initial particle distribution in space. This eliminates one possible cause of the discrepancy. It is assumed that the other published results have corrected for meteor distances. If not, this could overestimate the number of faint meteors and raise the $r$-factor. Another possibility is that the magnitude loss factors are not modeled correctly in the simulation for apparent angular velocity and some camera slew tests through stellar fields would help quantify the actual loss numbers.

The model also produces a total meteoroid count in a given volume of space, which can be scaled to the actual counts seen by the camera and applied to all elevations. This in turn leads to a spatial density estimate of meteoroids above a given mass. The mass limit is set by the limiting magnitude of the sensing system, which was found to be +6.5 in the raw video imagery. Although imaging processing of the video through multiframe averaging, mean removal and contrast enhancement can lower this to at best +7.5 , the higher limiting magnitude was adopted for the simulation 
as the user processed the raw imagery without the benefit of image enhancement.

Scaling the initial simulation meteoroid count of one billion with $r$-factor of 1.8 to obtain the counts per square degree observed, resulted in a simulation density of $1,000,000$ particles over the period of observation. Since the period of observation covered 1:50:00-2:06:41 UT or 1,001 seconds, a Leonid simulation with particle velocities of $71 \mathrm{~km} / \mathrm{sec}$ would have a long axis length of 71,071 kilometers. The simulated cross section of the spatial volume was $4.36 \times 10^{6} \mathrm{~km}^{2}$, and thus the average spatial density of meteoroids that produced the observed flux was found to be 3.2 \pm 0.8 particles per million cubic kilometers.

Thus the average flux of the Leonids at storm peak was $0.80 \pm 0.20$ particles per square kilometer per hour. If we adopt the Lorentzian profile derived from the meteor counts in near real-time (Jenniskens et al., 1999; $2000 \mathrm{~b}$ ), it is possible to relate the mean flux to the flux at the peak. In that case, the peak flux would then be $0.80 \times 1.03=0.82 \pm 0.19$ particles per square kilometer per hour. This flux level is for particles with intrinsic magnitude brighter than $m_{v}=+6.5$, which is thought to be equivalent to a mass of greater than 22 micrograms (Verniani, 1973; Hughes, 1987).

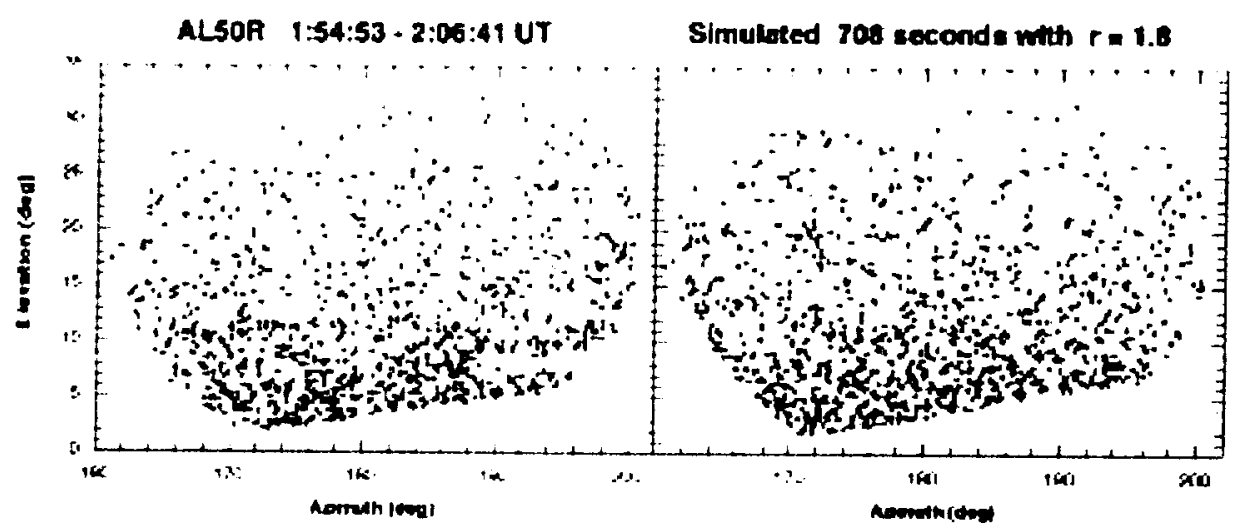

Figure 12. Simulated end point positions for the AL50R field of view (right) for a time duration that matches the measured data plot of figure 3 (left).

\subsection{Clustering ANALYSIS}

The calculated flux is computed as a mean over the observed area and several minutes of time. Examination of the videotape gives the impression there are waves meteors on short time scales and raises the question: is 
there any spatial or temporal correlation that show significant deviations from the mean flux?

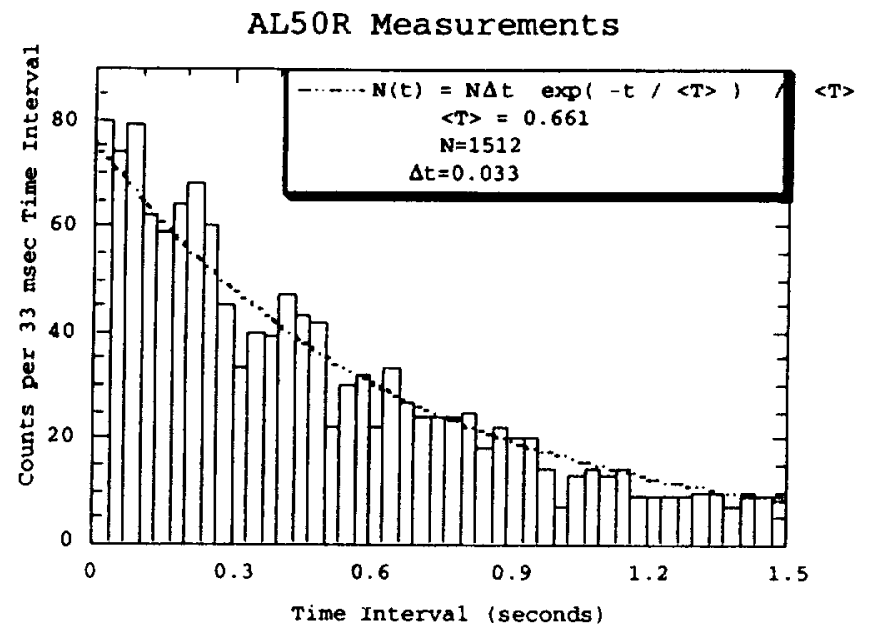

Figure 13. Histogram of time of arrival differences between successive meteors for the measured Leonids and a curve of the theoretical result for a spatially random meteoroid distribution.

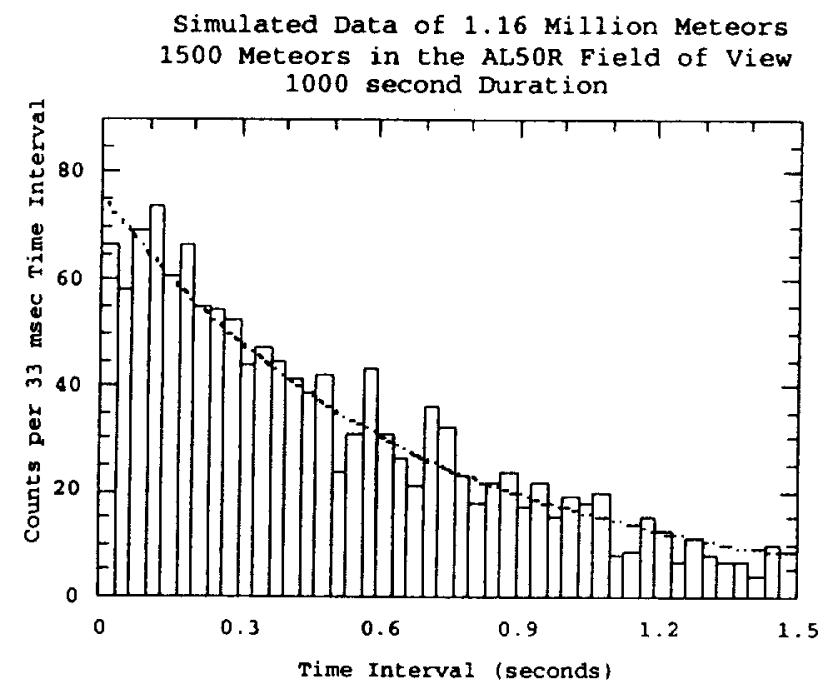

Figure 14. Histogram of time of arrival differences between successive meteors using the meteor simulation. 
The simulated flux level for the AL50R look direction of due south near the horizon compares favorably with the observed count distribution as seen by the AL50R camera (Figure 12). The simulation correctly predicts the strongest flux measurements will occur at very low elevation angles due to the lack of any appreciable atmospheric extinction at high sensor altitudes.

There is some apparent spatial correlation in the observed data that deserves further attention. The observed pattern (Figure 12, left) seems to show clusters and filaments on a spatial scale of about $50 \mathrm{~km}$ that are not apparent in the random simulated data (Figure 12, right). A more definitive answer on spatial clumping will need to await the analysis of more of the video record and a three dimension clustering evaluation.

Such spatial correlation can result from larger meteoroids that disintegrated at some point in the past. If such breakup would occur on approach of the Earth, one would expect temporal clustering of meteors on time scales less than 1 second. These are smaller time intervals than have been looked at before (e.g. Porubcan, 1968; Ofek, 1999). To address this issue, a histogram was made of the time difference between the brightest point in the temporally adjacent meteor tracks using all meteors in this analyzed video segment. Because of to the large range in meteor distances, the times of arrival were adjusted to reflect the change in position of the meteor end point along the radiant vector for the associated azimuth and elevation angles of each meteor.

Comparison of the video measurements to the simulated case and also to the theoretical curve for a random distribution following Porubcan (1968) and Ofek (1999), all show no deviation from a uniformly random distribution of meteoroids (Figure 13 and 14). At the temporal resolution of 66 milliseconds in the video imagery (5 kilometers spatially for the Leonids) there appears to be no clumping of meteors. We conclude that meteoroids do not tend to break up on approach to the Earth in the interplanetary medium or in the Earth's magnetosphere. Rather, the spatial correlation, if real, must result from breakup during a prior return of the comet. Even after a single evolution, each cluster of particles will tend to disperse like a dust trail and spread out rapidly prior to the time of arrival at the Earth. It might even be possible that such breakup occurs in the comet atmosphere shortly after ejection. In that case, temporal variations such as found in Figure 5, may signify the breakup of larger grains, and density correlations may exist over relatively large spatial scales.

\section{Optimal Pointing in Flux Measurements}

The remainder of this paper is concerned with the optimum viewing conditions for flux measurements that follow from the current simulation model, as well as variations in flux measurements that can occur as a result 
of pointing choices of camera fields. This discussion improves on earlier results in Jenniskens et al. $(1998 ; 1999$ a).
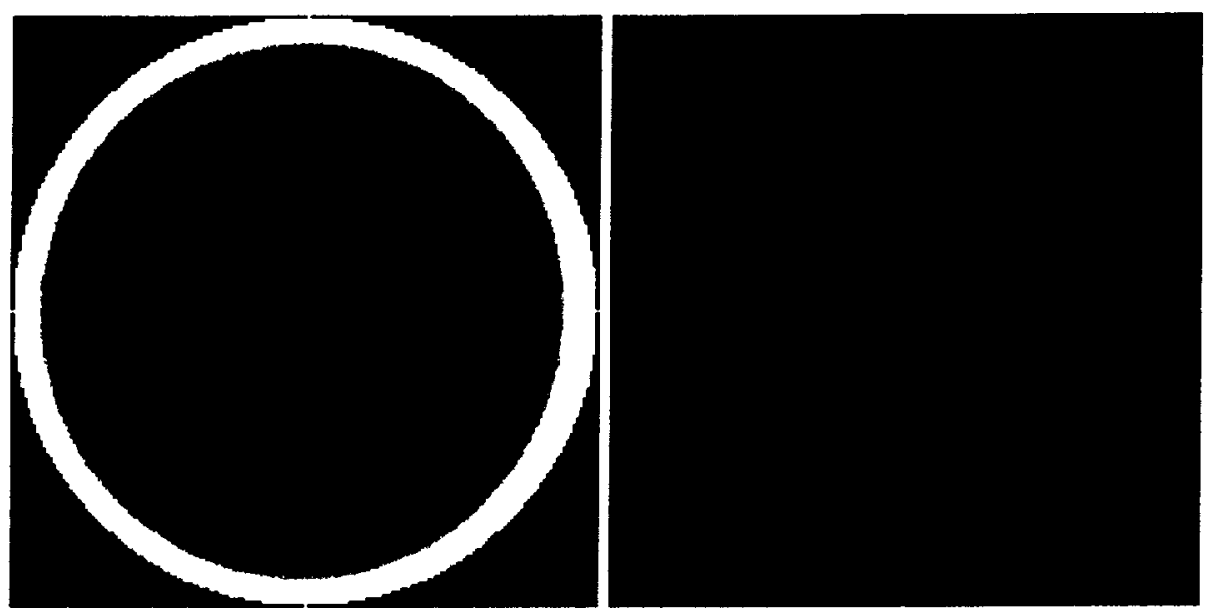

Figure 15 (left). Simulated all-sky flux levels for an airborne imager and $\mathrm{r}=1.8$. Figure 16 (right). As Figure 15, for a ground based imager.

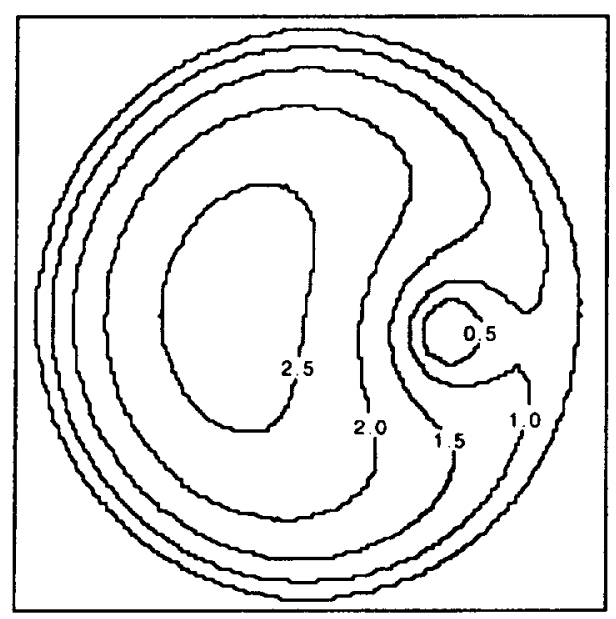

Figure 17. Magnitude losses due to the apparent velocity impact on integration time.

An illustration of the all-sky simulated flux levels for the night of the Leonid storm maximum is shown (Figure 15 and 16). The images are oriented with azimuth zero up, with azimuth measured positive in a clockwise fashion. The zenith is located dead center in the image with the 
horizon defined by the outer edge of the circular region. The radiant is located right of center. The simulated results are based on parameters at the time of peak flux for November 18, 1999 at $2 \mathrm{~h}$ UT. For the ARIA's position, eleven kilometers high over the Mediterranean Sea just south of Greece, the Leonid radiant was at an elevation of $48^{\circ}$ and an azimuth of $97^{\circ}$. The Leonids were assumed to have an end height of 95 kilometers. The camera's resolution of 3.7 arc minutes per pixel with 1/60 second integration time was determined from an earlier field of view calibration of the star imagery which also yielded a limiting magnitude of 6.5 .

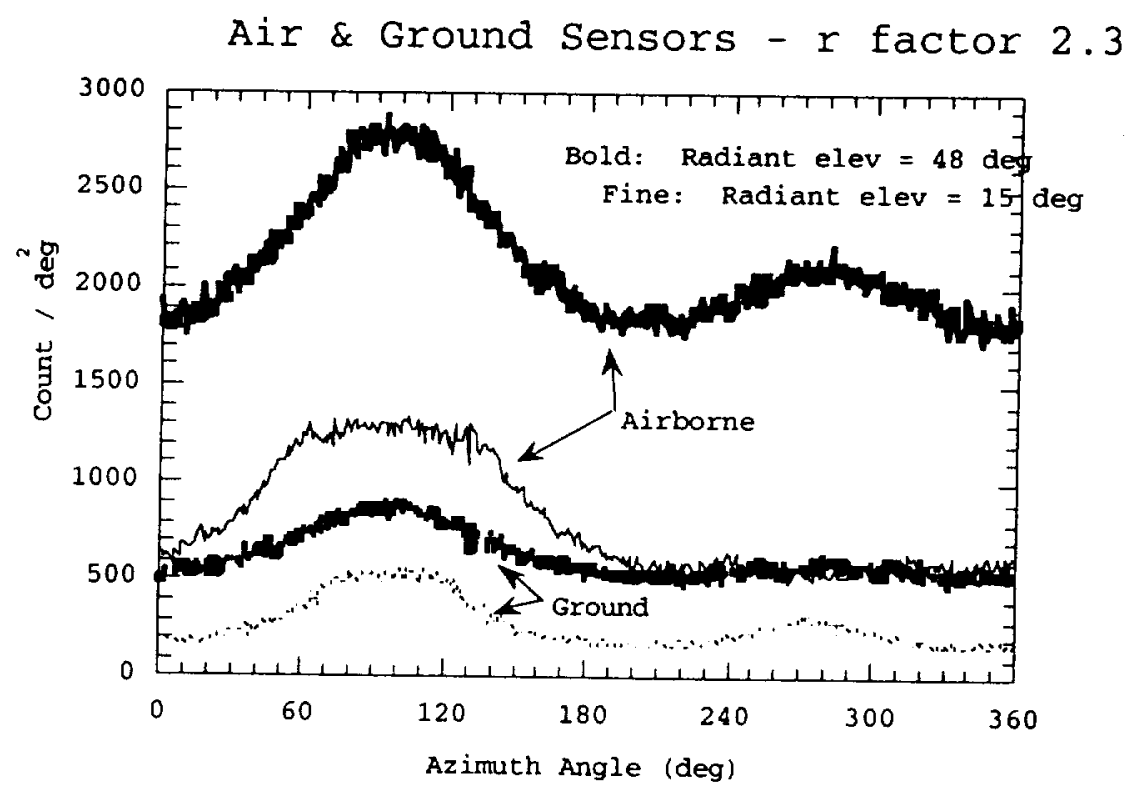

Figure 18. Azimuth cut of flux for air and ground based sensors for two different radiant elevation angles. These cuts were taken at the peak flux elevation of 3-4 degrees in the airborne case and 7-8 degrees in the ground case.

Comparing the airborne to ground flux simulation for a given radiant elevation and $r$-factor of $r=1.8$, there is at least a three-fold improvement in the number of meteors seen by flying at high altitudes and looking at low elevation angles (Figure 15 and 16). What also is interesting is that ground based CCD imagers are better off pointing at a low elevation towards the azimuth of the radiant to obtain the maximum number of meteors recorded. This is because the angular velocity is minimized (Figure 17). Another interesting feature in the all-sky flux plot is a hot spot 
of activity located at the radiant position. This feature is due to a one to two magnitude gain in detection of faint meteors from their slow apparent velocity in and around the radiant position of the otherwise fast moving Leonids. However, it has been remarked before that the very slow moving meteors near the radiant are not easily detected by a visual observer due to their highly foreshortened tracks, leading to a typical loss in counts for that look direction (Jenniskens, 1999a).

Next, several curves are presented to show the impact of radiant elevation and azimuth, sensor altitude, and limiting magnitude on a set of simulated flux counts (Figure 18 through 23). An elevation cut through the radiant and an azimuth cut near the maximum count elevation angle are presented in these figures extracted from an all-sky simulation result similar to figures 15 and 16. Unless otherwise indicated the results are for a meteor storm with $r$-factor of $r=2.3, a 71 \mathrm{~km} / \mathrm{sec}$ entry velocity, $95 \mathrm{~km}$ end height, radiant azimuth near 97 degrees, and a billion initial particles passing through an area $4.36 \times 10^{6} \mathrm{~km}^{2}$. The higher r-value is chosen to reflect values reported by ground-based observers (Brown et al., 2000).

Figure 18 illustrates the effect of azimuth of the pointing direction relative to the azimuth of the radiant. These cuts are for very low elevations representative of the peak counts computed. For the airborne case the elevation angles selected are a few degrees lower than the ground's peak elevation due to extinction effects. Note that the counts are integrated over one square degree and sensors with a wide field of view will have a different total count response when integrated over their associated viewing angles. In the southward direction of the AL50R sensor, the flux is relatively insensitive to azimuth but is at its lowest value because the angular velocity is at its highest (Figure 17). This was advantageous for our averaging the flux across the imager field of view but resulted in a loss of nearly $30 \%$ of the potentially observable meteors. In our model, the ground based system captures only $40 \%$ of those seen from the air for the same pointing direction.

Examining the elevation cuts passing through the radiant and 90 degrees from the radiant (Figure 19 and 20 ) shows the advantage again of pointing in the direction of the radiant's azimuth. The airborne based sensor clearly shows at least a three-fold improvement over ground based measurements in observed flux levels in the low elevation directions. This gain is because below the radiant, the angular velocity is lower than elsewhere. The angular velocity is very low near the radiant also, but that does not necessary lead to higher meteor counts (Jenniskens et al., 1998). Typically, the point meteors are not easily identified in the noisy background of the intensified video cameras. The effect is clearer in photographic data, where the radiant is an efficient area for detecting meteors. For flux measurements, the radiant is not a good location because the angular velocity changes rapidly with location in the field of view. That makes the result extremely sensitive to detector properties. 


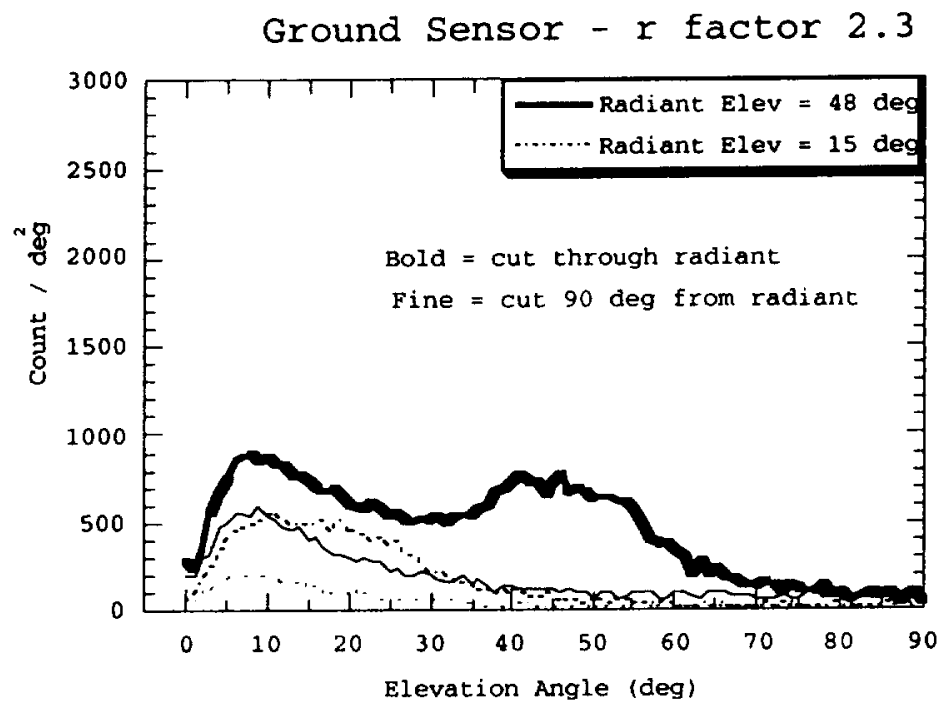

Figure 19. Elevation cut of flux for a ground-based sensor for two different radiant elevation angles. The bold lines at elevation cuts that pass through the radiant. The fine lines are elevation cuts for an azimuth ninety degrees from the azimuth of the radiant.

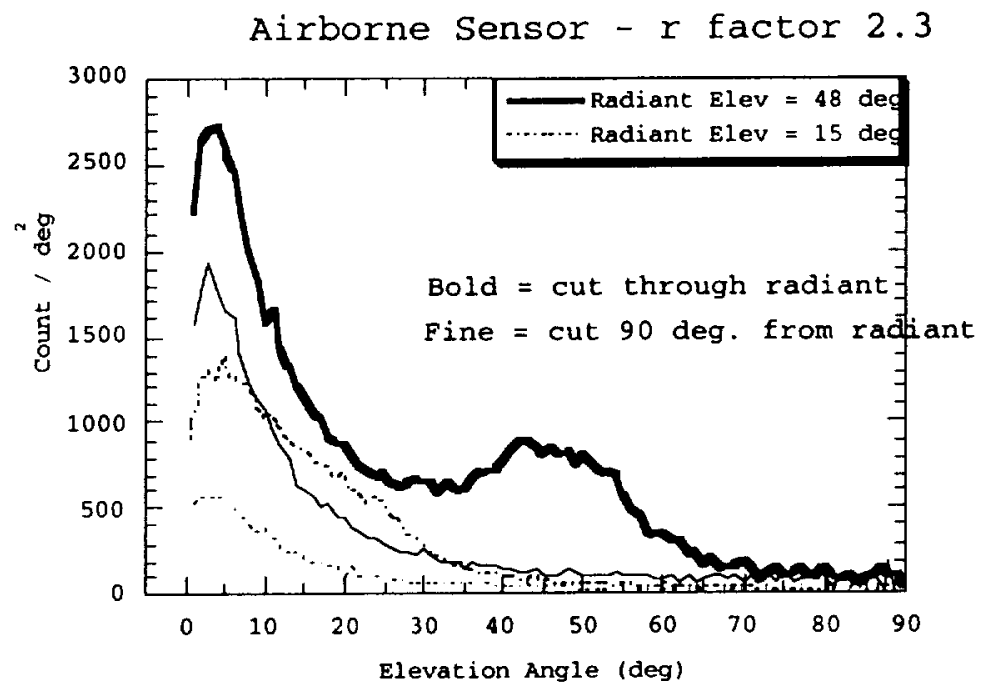

Figure 20. Elevation cut of flux for an airborne-based sensor for two different radiant elevation angles. The bold lines at elevation cuts that pass through the radiant. The fine lines are elevation cuts for an azimuth ninety degrees from the azimuth of the radiant. 
The impact of $\mathrm{r}$-factor on the air and ground results shows similarity with one another (Figures 21 and 22). Above 40 degrees elevation there is no $r$ factor dependence on total flux observed. Only below 40 degrees can the combined effects of extinction, distance, and speed begin to spread the curves as more of the fainter parts of the distribution are missed due to the increasing magnitude losses. Finally not until very low elevation angles does the airborne flux levels soar due the dominating effects of extinction on the ground. If the shower is dominated by fainter particles and a high $r$ factor then it appears prudent to point the imager at somewhat higher elevation, as noted before (Jenniskens et al., 1998).

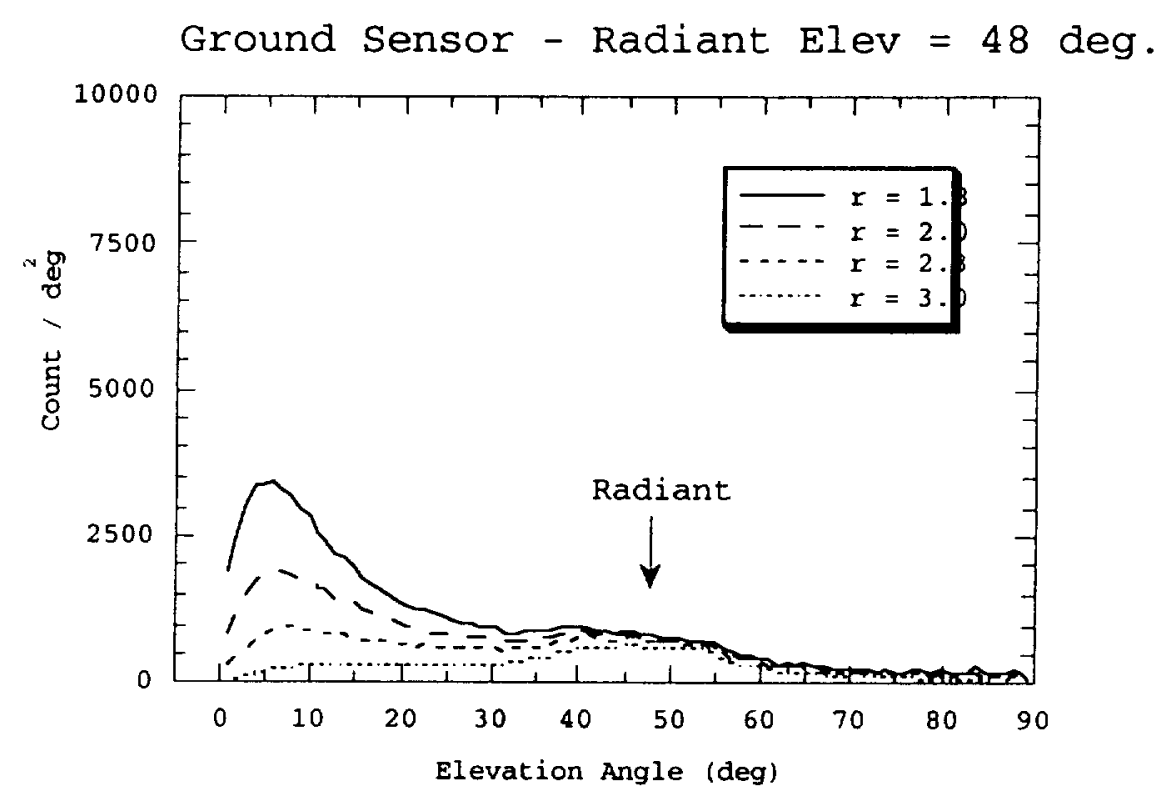

Figure 2I. Elevation cut of flux for a ground sensor for different $\mathrm{r}$ factors.

Finally, the impact of limiting magnitude was examined, because for the 2000 and 2002 Leonids, there will be the interfering effects of moonlight (Figure 23). For the airborne case, the presumption is that the limiting magnitude drop may amount to no more than 0.2 magn., assuming a look direction away from the moon's position in the sky. For the ground case however, the losses would be 0.5 magn. or perhaps as high as 1.0 magn. for non-ideal conditions. The impact is to lower the apparent ground measured fluxes by 30 to 60 percent. Without better understanding of the effects of light scattering on limiting magnitudes at various elevations, the effect in the model is simply a scale factor on the flux in a given direction. 


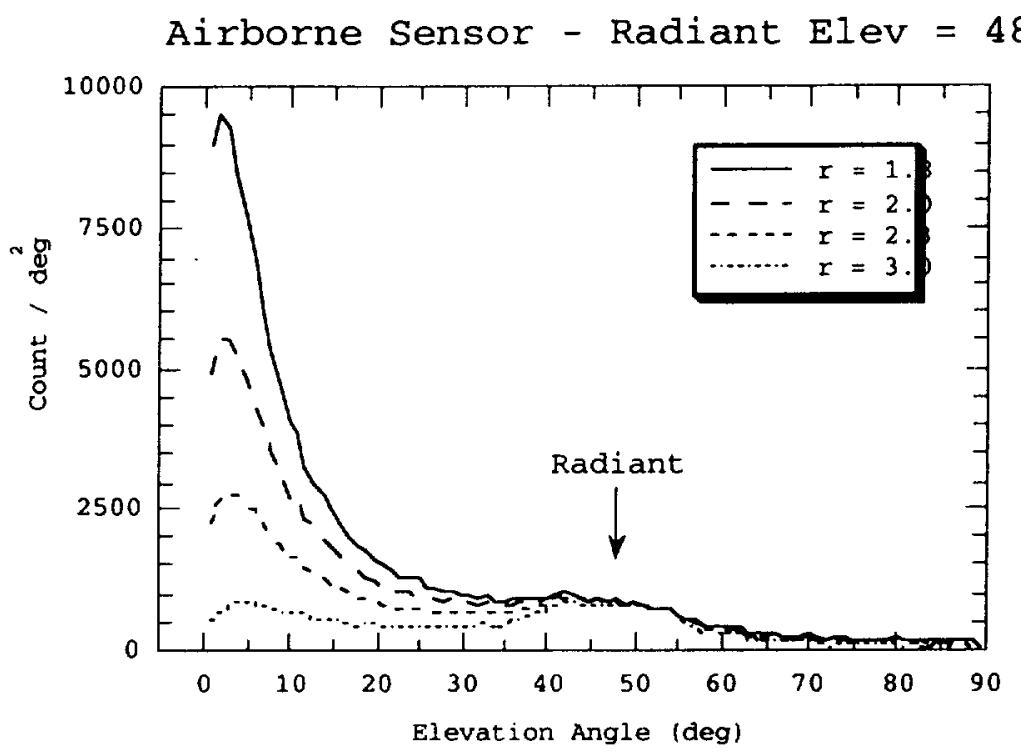

Figure 22. Elevation cut of flux for an airborne sensor for different $\mathbf{r}$ factors.

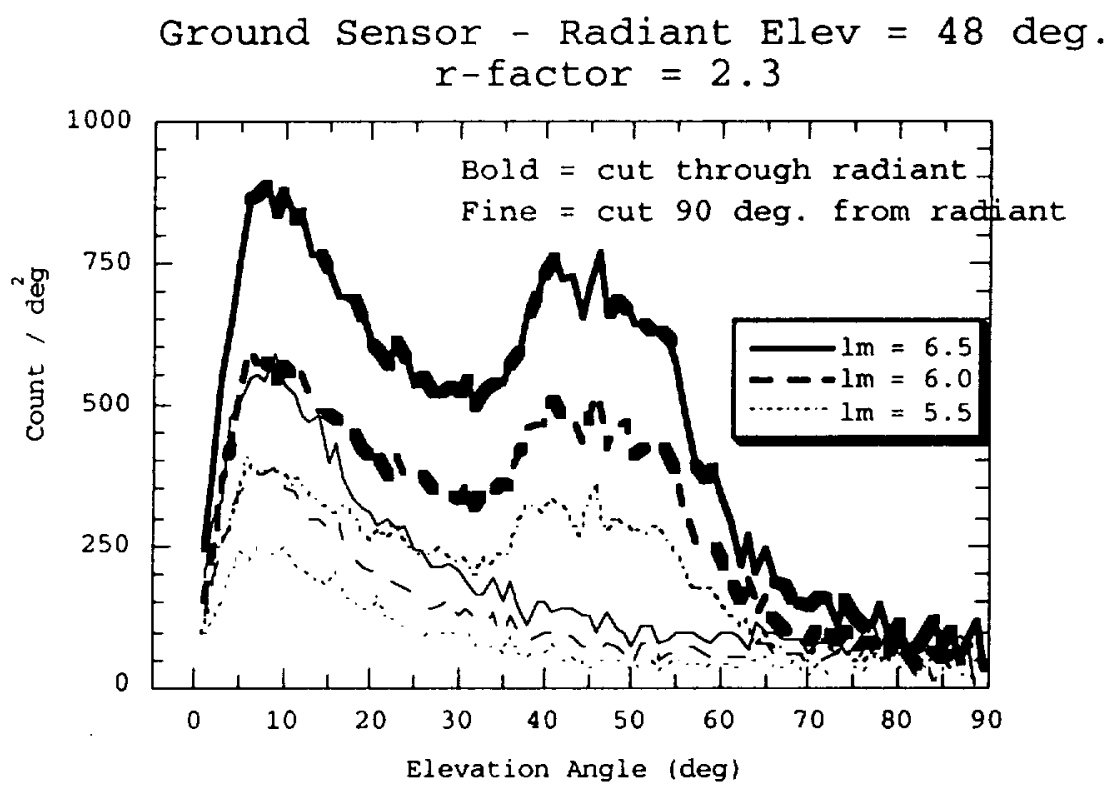

Figure 23. Elevation cut of flux for a ground-based sensor for different limiting magnitudes. 
In conclusion, overall pointing recommendations for video sensors in order to maximize measured flux seem equivalent for air and ground based systems. Perpendicular to the radiant at low elevation provides highest counts and uniform conditions across the field of view. Yet higher rates are observed towards the azimuth of the radiant at low elevation angles. However, poor detectability of short meteor trails and a rapidly changing angular velocity with location in the field of view, makes the results from such observations extremely sensitive to detector properties.

In order to improve our understanding of the instrument properties in relation to observing geometry and observing conditions, it is best to do simultaneous measurements with multiple cameras at different azimuth and elevation angles and compare the results with a model as described in this paper. Koschny and Zender (2000) have published the first effort at this approach.

Until now, only a small fraction of videotaped data has been analyzed. Future work will concentrate on the possible spatial and temporal correlation detected in this paper. Of particular interest is the information that may be retrieved about the ejection and subsequent breakup of large meteoroids. In addition, the concentration of meteors near the horizon is still a surprise in our opinion. An unlikely low r-value was needed to provide a realistic distribution of meteors in the simulation model. Hence, the spatial model of meteor rates on the sky needs to be further improved to account for the observed increase of meteor rates near the horizon and improve the absolute calibration of the meteor influx.

\section{Acknowledgments}

During the meteor storm, Mr. Gural was a member of the global ground flux monitoring team lead by Dr. Peter Brown, but unfortunately was clouded out in the Canary Islands during the peak flux. He is most grateful for having been able to witness and contribute to the analysis of this once in a lifetime meteoric event from videotape. This furthers the goals of the Pro-Amat Working Group of IAU Commission 22. The work for the 1999 Leonid Multi-Instrument Aircraft Campaign was sponsored by NASA's Planetary Astronomy and Atmospheres Program. Leonid MAC was sponsored by NASA's Planetary Astronomy, Exobiology, Sub-orbital MITM, and Advanced Missions and Technologies for Astrobiology programs, NASA Ames Research Center, and the U.S. Air Force/XOR. The ARIA aircraft's operational support was provided by the USAF/452 ${ }^{\text {nd }}$ Flight Test Squadron. Editorial handling: Noah Brosch. 


\section{References}

Arlt, R.: 1998, WGN, Journal of the IMO 26, 239--248

Arlt, R., Rubio, L.B., Brown, P., and Gijssens, M.: 1999, WGN, Journal of the IMO 27, 286--295.

Brown, P., Campbell, M.D., Ellis, K.J., Hawkes, R.L., Jones, J., Gural, P., Babcock, D., Barnbaum, C., Bartlett, R.K., Bedard, M., Bedient, J., Beech, M., Brosch, N., Clifton, S., Connors, M., Cook, B., Goetz, P., Gaines, J.K., Gramer, L., Gray, J., Hildebrand, A.R., Jewell, D., Jones, A., Leake, M., LeBlanc, A.G., Looper, J.K., McIntosh, B.A., Montague, T., Morrow, M.J., Murray, I.S., Nikolova, S., Robichaud, J., Spondor, R., Talarico, J., Theijsmeijer, C., Tilton, B., Treu, M., Vachon, C., Webster, A.R., Weryk, R., and Worden, S.P.: 2000, Earth, Moon and Planets 82-83, 167-190.

Gural, P.: 1999a, MeteorScan Documentation and User's Guide, Version 2.3, Published by Peter Gural, Sterling, Virginia, $120 \mathrm{pp}$.

Gural, P.: 1999b, WGN, Journal of the IMO 27, 111--114.

Hughes, D.W.: 1987, Astron. Astrophys. 187, 879--888.

Jenniskens, P.: 1999a, Meteoritics Planet. Sci. 34, 959--968.

Jenniskens, P.: 1999b, Adv. Space Res. 23, 137-147.

Jenniskens, P. and Butow S.J.: 1999, Meteoritics Planet. Sci. 34, 933--943.

Jenniskens, P., de Lignie, M., Betlem, H., Borovicka, J., Laux, C.O., Packan, D., and Krüger, C.H.: 1998, Earth Moon and Planets 80, 311--341.

Jenniskens, P., Crawford, C., and Butow, S.: 1999, WGN , Joumal of the IMO 28, 58--36.

Jenniskens, P., Butow, S.J., and Fonda, M.: 2000a, Earth, Moon and Planets 82-83, $1-26$.

Jenniskens, P., Crawford, C., Butow, S.J., Nugent, D., Koop, M., Holman, D., Houston, J., Jobse, K., Kronk, G., and Beatty, K.: 2000b, Earth, Moon and Planets 82-83, 191-208.

Koschack, R. and Rendtel, J.: 1990a, WGN, Journal of the IMO 18, 44--58.

Koschack, R. and Rendtel, J.: 1990b, WGN, Journal of the IMO 18, 119--140.

Koschny, D. and Zender, J.: 2000, Earth, Moon and Planets 82-83, 209-220.

Ofek, E.O.: 1999, WGN, Journal of the IMO 27, 172--176.

Porubcan, V.: 1968, Bull. Astron. Inst. Czechoslov. 19, 316--323.

Roth, G. D.: 1994, Compendium of Practical Astronomy, Volume 3, Stars and Stellar Systems, Springer-Verlag, Berlin, 321 pp.

Steyaert, C: 1990, IMO Monograph No. I, Photographic Astrometry, Published by the Computer Commission of the International Meteor Organization, $59 \mathrm{pp}$.

Van der Veen, P.: 1986b, Radiant, Journal of the Dutch Meteor Society 6, 41--45.

Verniani, F.: 1973, J. Geophys. Res. 78, 8429--8462. 